\title{
"Chronic Recurrent Abdominal Pain And Evaluation of Disease By Various Diagnostic Modalities"
}

\author{
Dr. A. Balamurugan M.S (General Surgery) \\ Assistant Professor, Dept Of General Surgery Coimbatore Medical College Hospital Coimbatore
}

\begin{abstract}
Chronic abdominal pain is defined as constant or intermittent pain persisting for more than 3 months. It presents a diagnostic and treatment challenge to the general surgeons and it remains a common surgical problem. This study is done mainly in arriving the diagnosis of disease, by using various diagnostic modalities and to highlight the reliable investigation for various diseases causing chronic pain abdomen. The common etiologies for chronic recurrent pain abdomen in our hospital are Peptic ulcer, chronic pancreatitis, Gastritis, GERD, TB abdomen followed by malignancy. Endoscopy and diagnostic laparoscopy are found to be useful in the diagnosis of upper and lower abdominal pathologies respectively. Conservative management was found to be successful in the treatment of patients with peptic ulcer disease and chronic pancreatitis. A subgroup of patients had chronic intractable abdominal pain were referred to psychiatrist for further management.
\end{abstract}

Keywords: Abdominal pain, alcohol, diagnostic laparoscopy, peptic ulcer, smoking

\section{Introduction}

Chronic abdominal pain is defined as constant or intermittent pain persisting for more than 3 months. Here intermittent pain may be referred to as recurrent abdominal pain. In between the periods of pain, patient remains asymptomatic. It presents a diagnostic and treatment challenge to the general surgeons and it remains a common surgical problem. Specific diagnosis could not be arrived despite all necessary investigations. The cost of health care is high, due to need for sophisticated investigations and the treatment.

This study is done mainly in arriving the diagnosis of disease, by using various diagnostic modalities available at Coimbatore medical college hospital that is X-ray, USG abdomen, plain and contrast enhanced CT, contrast studies, colonoscopy, MRI, OGD scopy, Diagnostic laparoscopy and all other investigations and to study which will be the reliable investigation for various diseases causing chronic pain abdomen and to arrive at a diagnosis.

\section{Aims And Objectives}

To identify the common etiologies for chronic recurrent pain

To make a definitive diagnosis by using various diagnostic modalities available at Coimbatore Medical College Hospital

To give effective relief to the patient.

\section{Materials And Methods}

Time period of study:- September 2011 to November 2012

Age Of Patients -Older Than 13years

Gender Of Patients - Male And Female

Study Area -Coimbatore Medical College Hospital

Study Population-Patients presenting to the surgical department with features of chronic recurrent pain abdomen

3.1. Selection Criteria

3.1.1.Inclusion Criteria

- Chronic recurrent pain abdomen

- $\quad$ Age more than 13years

\subsubsection{Exclusion Criteria}

- Acute pain abdomen

- Patients treated by psychiatrists

- Coagulopathy, severe cardiopulmonary disease

- Previous surgeries

- Urological and gynecological causes 


\subsection{Study Design}

Prospective observational study was conducted on patients admitted with chronic recurrent pain abdomen

\subsection{Parameters Studied}

- Age

- Sex

- Dietary pattern

- Smoking pattern

- Socio economic status

- Type of pain

- Location of pain

- Diagnostic investigation which was confirmatory

- Diagnosis

- Treatment

- Out come

\section{1age Wise Distribution}

\section{Results And Observation}

Patients in the age group 31-40 years are commonly affected with a mean age of 43.64 years.

\begin{tabular}{|l|l|l|}
\hline Age Group & No of Patients & Percentage \\
\hline $11-20$ & 1 & 2 \\
\hline $21-30$ & 9 & 18 \\
\hline $31-40$ & 16 & 32 \\
\hline $41-50$ & 9 & 18 \\
\hline $51-60$ & 6 & 12 \\
\hline $61-70$ & 8 & 16 \\
\hline $71-80$ & 1 & 2 \\
\hline
\end{tabular}

\section{2smoking Pattern}

$64 \%$ of the study group was smokers as compared to the $36 \%$ non-smokers. This may suggest that smoking may have a positive correlation with chronic abdominal pain of certain causes

\section{3alcohol Intake Pattern}

$62 \%$ of our patients were consumers of alcohol and of these, $32 \%$ had consumed for more than 10 years. Hence alcohol may also act as an additive factor in many diseases presenting as chronic abdominal pain.

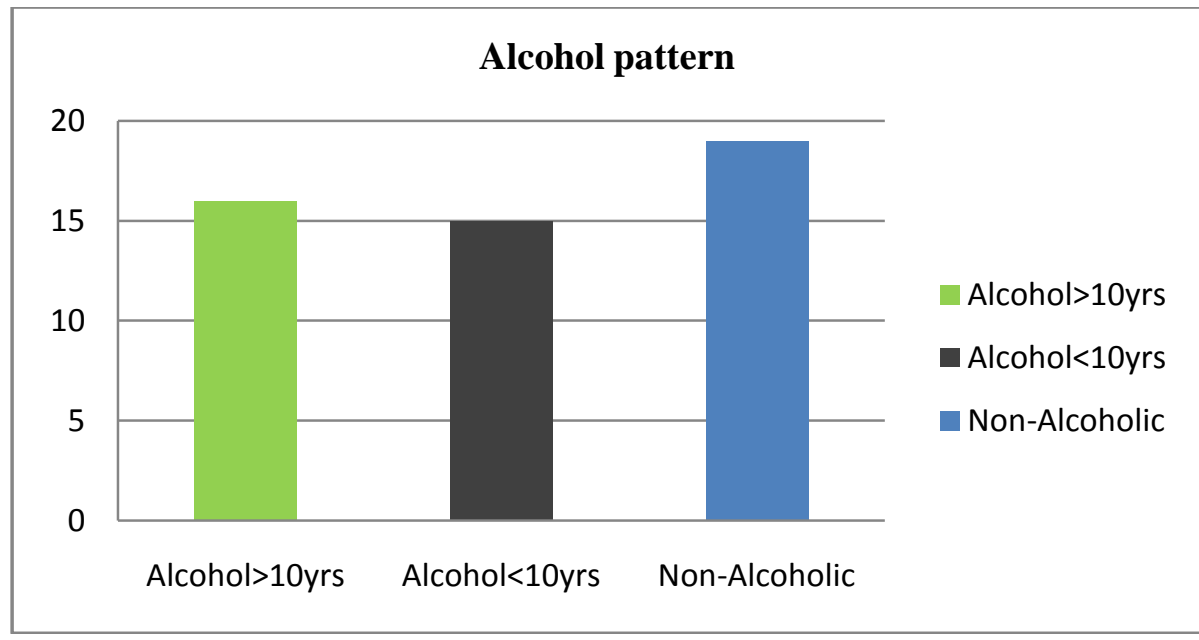

\subsection{Dietary Pattern}

$72 \%$ of the patients consumed non-vegetarian food which suggests that non-vegetarian food may be a predisposing factor in diseases causing chronic abdominal pain 


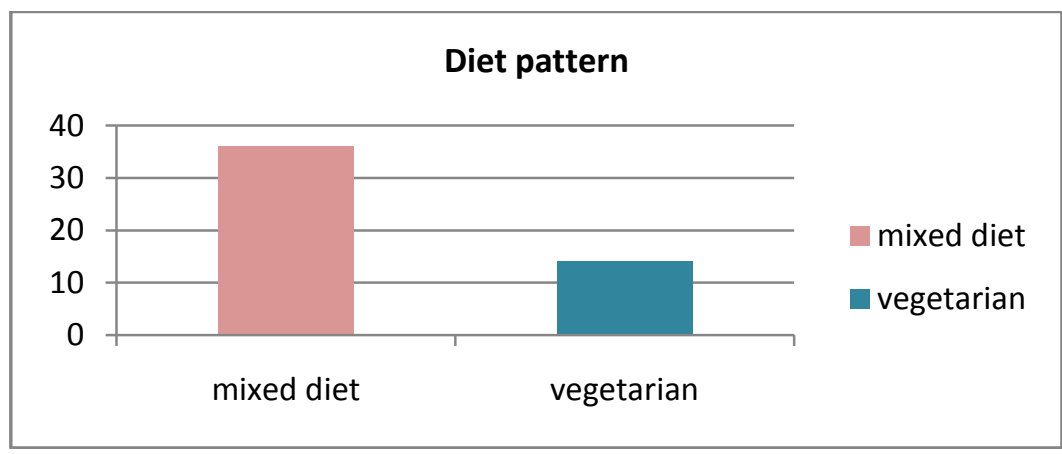

\section{5habit Of Betel Nut Chewing}

$38 \%$ of patients were betelnut chewers chronically and more among this group was female individuals.

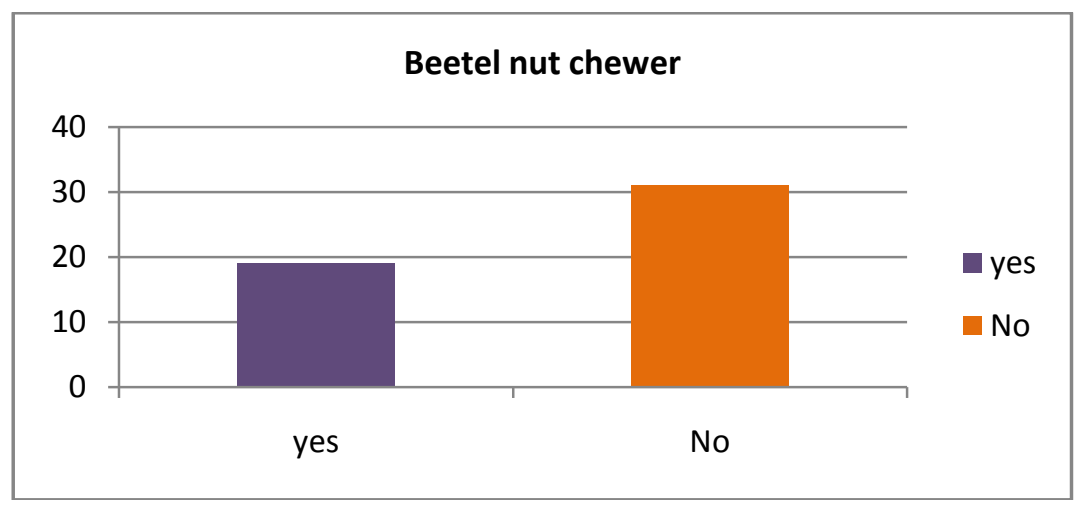

\section{6socio Economic Status}

Being a government run tertiary care institution, all the patients that were observed in the study were from a lower socio-economic class.

\section{7type Of Pain}

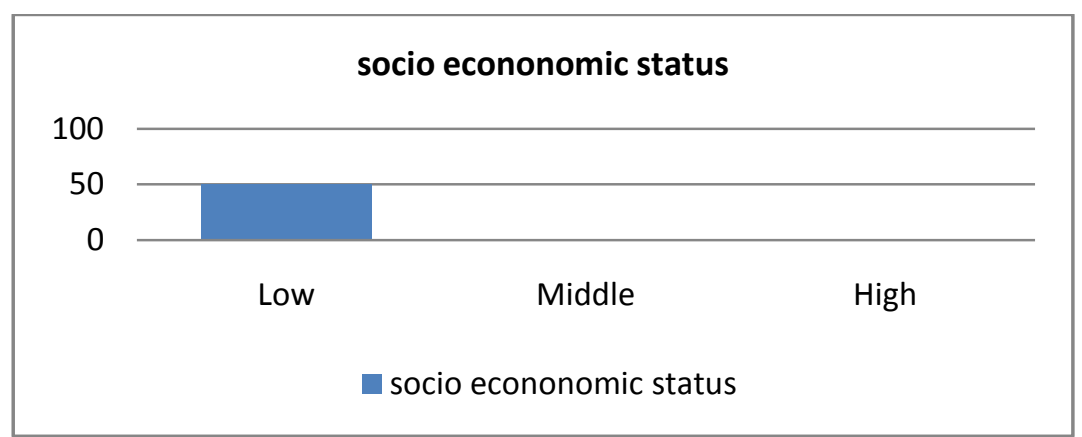

Most of the patients presented with dull aching pain, burning pain and small number of patients with colicky pain abdomen.

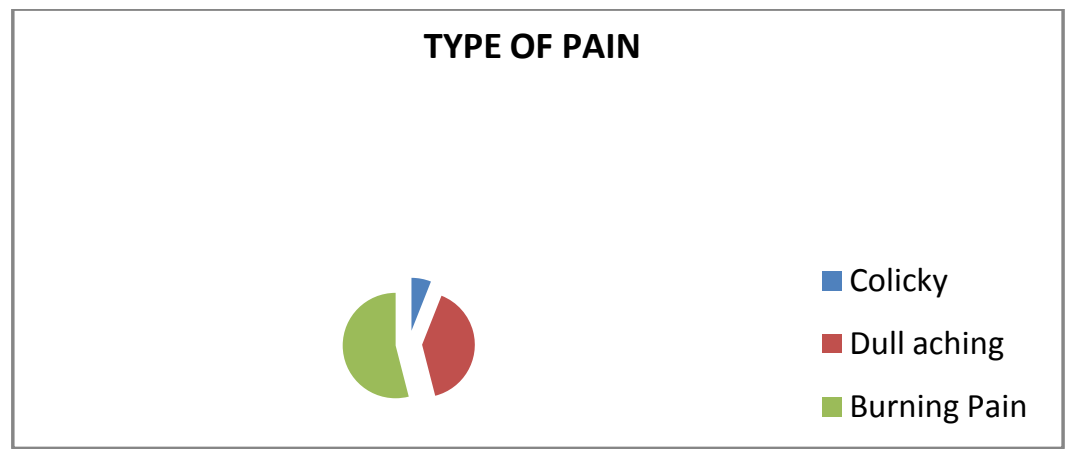




\section{8site Of Pain}

$64 \%$ of patients complained of epigastric pain followed by Right upper quadrant (16\%), right lower quadrant (10\%) and left iliac fossa (10\%).

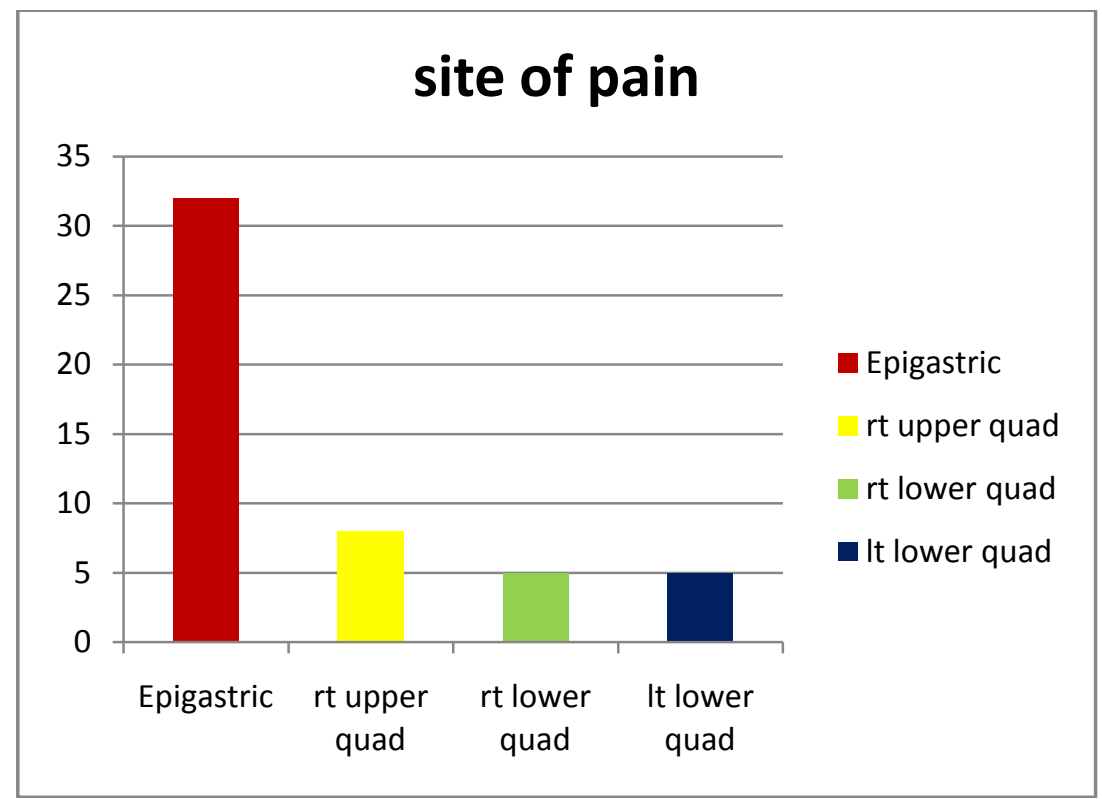

\subsection{Diagnostic Investigation Which Was Confirmatory}

OGD scopy revealed the diagnosis in most patients with GERD, peptic ulcer disease and gastritis. CT was more useful than ultrasonography in cases of pancreatitis. TB abdomen was most tough in arriving at a definitive diagnosis and this was done using ultrasonography, CT scan and diagnostic laparoscopy. Colonoscopy proved its importance in intraluminal pathologies such as neoplastic growths.

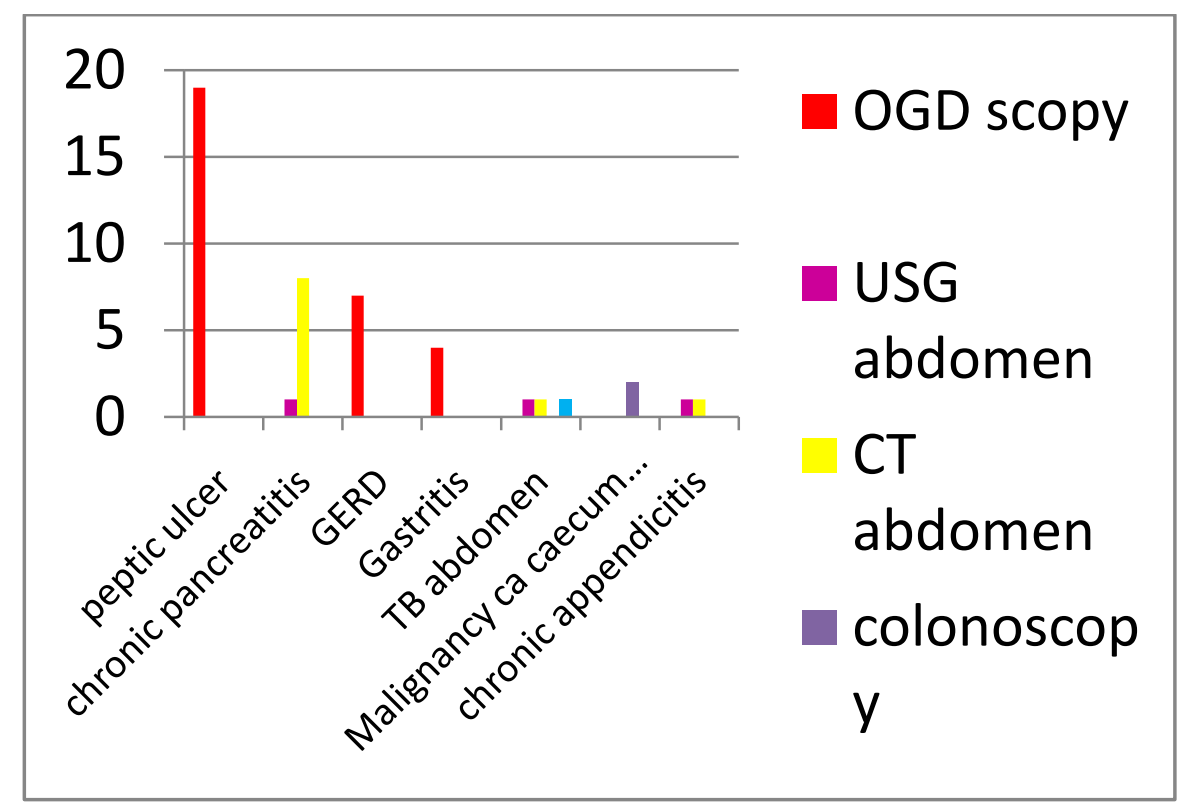

\subsection{0etiology In Males}

Peptic ulcer was the common disease identified followed in order by chronic pancreatitis, GERD,gastritis followed by TB abdomen.The percentage of undiagnosed patients was $6 \%$. 


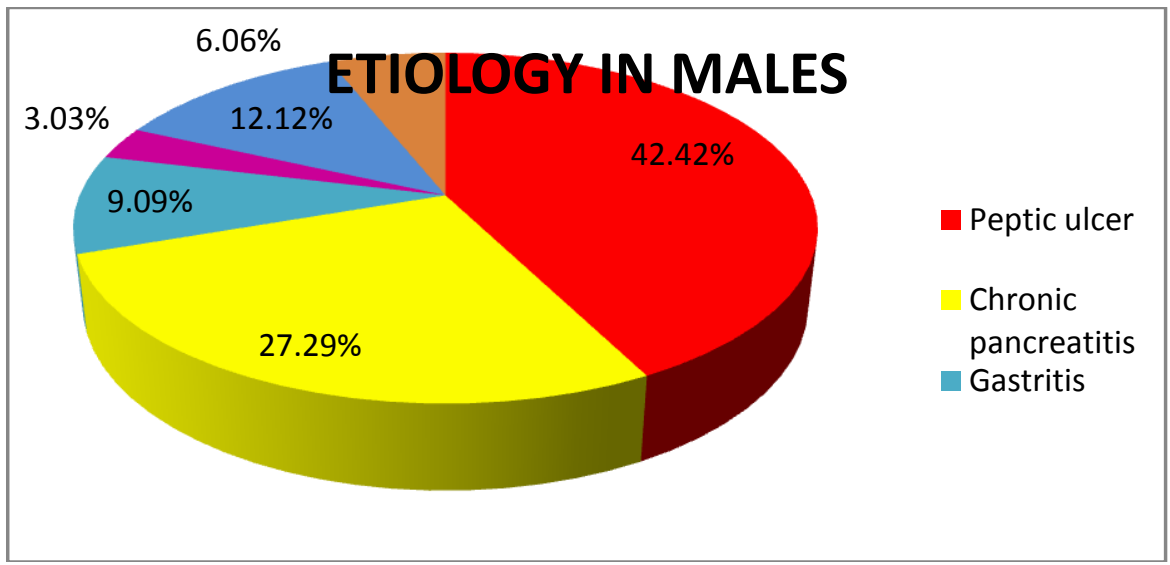

\section{ETIOLOGY IN FEMALES}

\section{$17.64 \%$}

$11.76 \%$

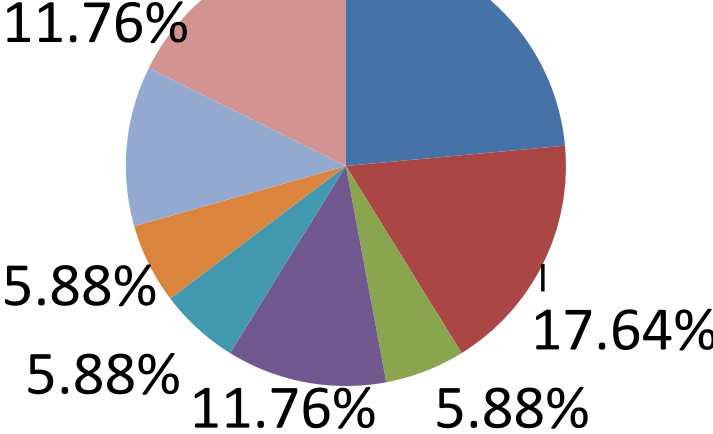

\subsection{1conservative Management}

$40 \%$ of the patients were treated by Triple $\mathrm{H}$ therapy for 2 weeks, $22.22 \%$ patients were treated with proton pump inhibitors most of them diagnosed to have GERD, 4.44\% of patients treated with 6 months duration of anti tuberculous therapy, patient with chronic pancreatitis treated by simple analgesics and finally, placebo was given to undiagnosed chronic pain abdomen.

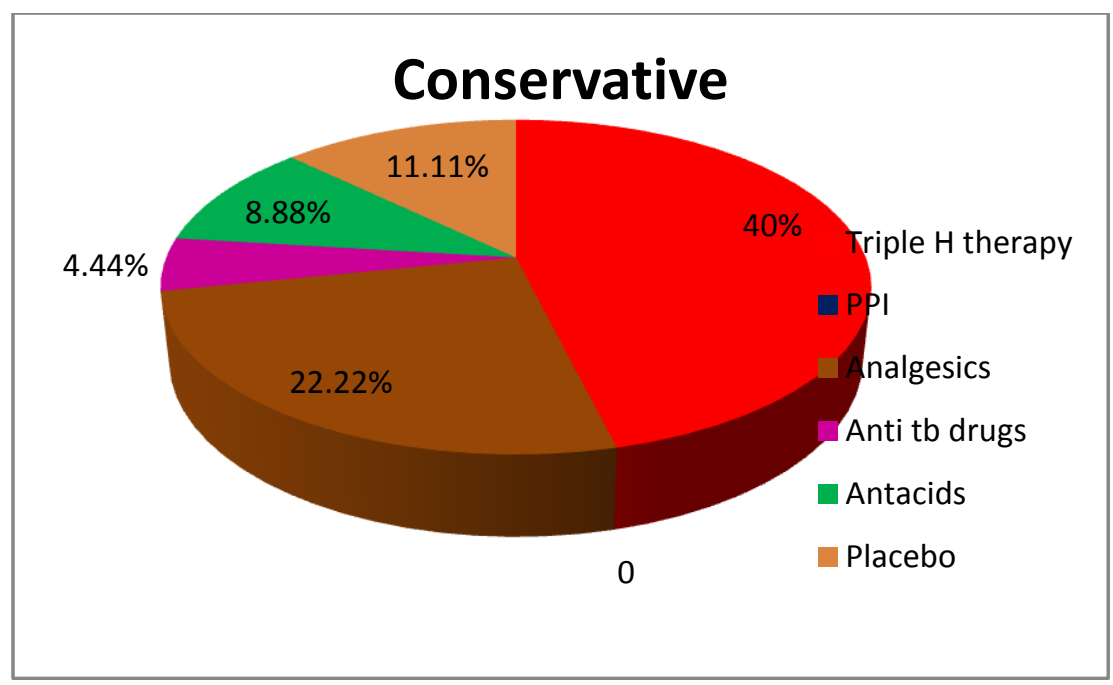




\subsection{2surgical Treatment}

5 patients were surgically treated, out of which Right Hemi colectomy was done to two patients with carcinoma caecum and ascendingcolon. Laparoscopic appendicectomy was done for one patient for chronic appendicitis. One patient suspected to have TB abdomen omental biopsy was done and for one patient with chronic pancreatitis celiac ganglionic block was done.

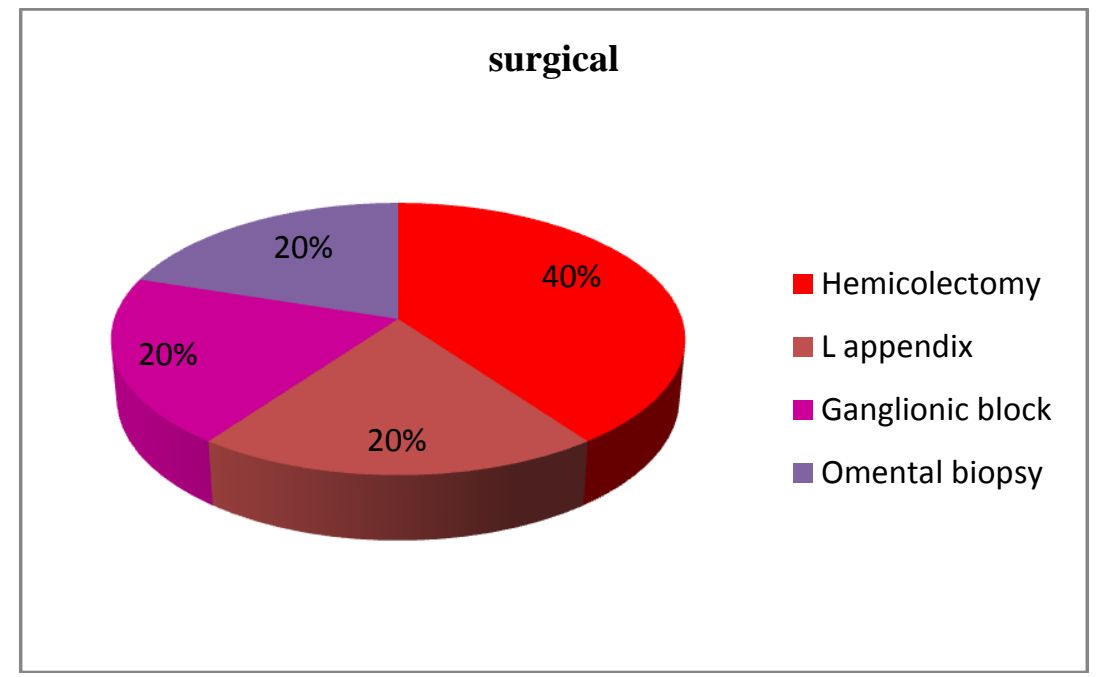

\subsection{3treatment And Outcome}

Out of 50 patients, 45 patients were treated conservatively, of which 25 patients were relieved of their symptoms. And out of the 5 patients treated surgically 4 patients got relieved of their symptoms.

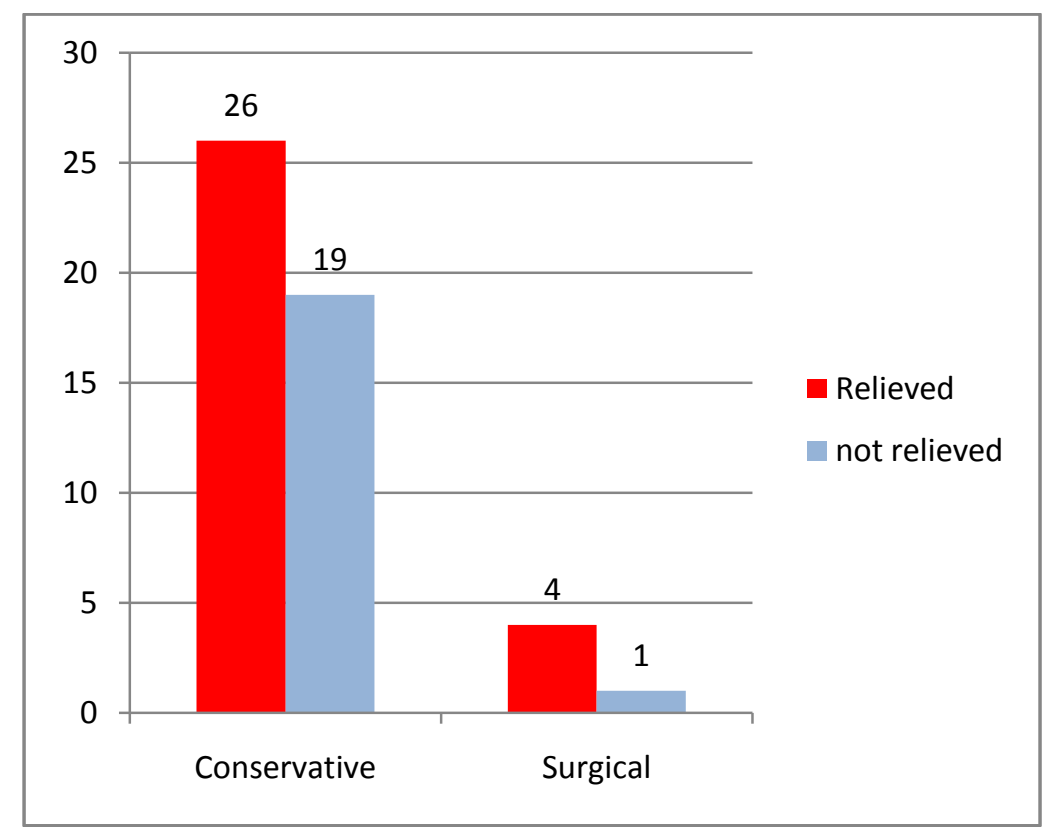

\section{Conclusion}

1. The common etiologies for chronic recurrent pain abdomen in our hospital are Peptic ulcer, chronicpancreatitis, Gastritis, GERD, TB abdomen followed by malignancy.

2. For patients with upper abdominal pathology, endoscopy proves to be a confirmatory diagnosis, and for patients with lower abdominal pathology CT abdomen and Diagnostic laparoscopy gives better information in arriving at a diagnosis.

3. $\mathrm{X}$ rays do not pick up any confirmatory diagnosis in the diagnosis of chronic recurrent pain abdomen.

4. Peptic ulcer patients were treated by conservative management and did not develop any surgical complications. On 3 months follow up, 8 patients were relieved of their symptoms and at 6 months follow up 8 patients were relieved of their symptoms. 
5. For patients with chronic pancreatitis symptomatic pain relief was given to the patient, however general condition of the patient could not be improved. Most of the patients were treated conservatively. On follow up at 3 and 6 months only one patient who had undergone celiac ganglionic blockade got relieved of his symptoms and rest of the patients were not relieved of their symptoms

6. For 5 patients of which 3 are females and 2 are males for whom definitive diagnosis could not be arrived at inspite of all investigations hence these patients were classified as chronic intractable abdominal pain, found to be more common in females, they were given placebo referred to psychiatrists for further management.

[1]. Short Practice of Surgery, Bailey \& Love, $25^{\text {th }}$ ed.

\section{Bibliography}

[2]. Textbook of surgery, Sabiston, $18^{\text {th }}$ ed

[3]. Oxoford textbook of surgery,J. Morris and William C Wood, $2^{\text {nd }}$ ed, vol 3

[4]. Mastery of Surgery, Joseph E Fisher, $5^{\text {th }}$ ed. 